\title{
Great Awakening - Telerehabilitation in Physiotherapy during Pandemic and Impact of COVID-19
}

\author{
Shrushti Prashant Jachak ${ }^{1}$, Pratik Arun Phansopkar², Waqar Mohsin Naqvi³, Kiran Kumar ${ }^{4}$ \\ 1,2,4 Department of Musculoskeletal Physiotherapy, Ravi Nair Physiotherapy College, \\ Datta Meghe Institute of Medical Sciences, Wardha, Maharashtra, India. ${ }^{3}$ Department of \\ Community Physiotherapy, Ravi Nair Physiotherapy College, Datta Meghe Institute of \\ Medical Sciences, Wardha, Maharashtra, India
}

\section{ABSTRACT}

Coronavirus disease in 2019, also called COVID-19 which has spread worldwide has given rise to a pandemic situation. The aetiological agent is the (SARS-CoV-2) the Severe Acute Respiratory Syndrome Coronavirus 2. The COVID-19 pandemic has an unusual way of striking the entire world. Crises has spread rapidly. Disease load and casualties are still on the rise, and crisis influence is spreading across developing countries. Around the globe, the reactions, perceptions, and outcomes were distinct. The outbreak has reflected unfavourable mental health impacts and symptoms. This pandemic has also affected the healthcare department that is treating the patients suffering from diseases other than corona. The power and severity of slowdown varying from being temporary to a long-term recession, they are unanimous about the fact that the slowdown would have an intense impact amongst various sectors of the economy. Most importantly, some panic among consumers and firms has disfigured normal patterns of consumption and caused market inconsistency. Digitalization and automation have proved to be the solution in this challenging phase. Telemedicine and Tele physiotherapy for online consultancy have changed the time in a positive way. Conducting live sessions reduces the psychological risks to the patients and also is beneficial for the motivation of the therapy. Tele-rehabilitation increases the level of treatment for people with disorders in both physical and mental wellbeing, lowers hospital expenses, and strengthens conventional face-to-face practice. This article would give the overall idea of how the social, economic, cultural, and psychological aspects are influenced by the pandemic.

\section{KEY WORDS}

COVID-19, Telerehabilitation, Pandemic, Physiotherapy, Health Care, Economy
Corresponding Author: Dr. Pratik Arun Phansopkar. Assistant Professor, Department of Musculoskeletal Physiotherapy, Ravi Nair Physiotherapy College, Datta Meghe Institute of Medical Sciences, Wardha, Maharashtra, India. E-mail: drpratik77@gmail.com

DOI: $10.14260 /$ jemds/2020/744

How to Cite This Article: Jachak SP, Phansopkar PA, Naqvi WM, et al. Great awakening - telerehabilitation in physiotherapy during pandemic and impact of COVID 19. J Evolution Med Dent Sci 2020;9(45):3387-3393, $10.14260 /$ jemds $/ 2020 / 744$

Submission 06-07-2020,

Peer Review 07-09-2020,

Acceptance 14-09-2020,

Published 09-11-2020.

Copyright (C) 2020 Pratik Arun Phansopkar et al. This is an open access article distributed under Creative Commons Attribution License [Attribution 4.0 International (CC BY 4.0)] 


\section{BACKGROUND}

The 2019 corona virus pandemic is a public health emergency of global concern rising provocations of many economical and psychological issues. Low and middle income countries are rushing to respond to this massive and fast moving challenge. The effects of the disease on mortality and morbidity has become important in a highly industrialized environment since the outbreak. Because of the global economy stagnation with production intervention, the workings of global supply chains have been disrupted. Companies all over the world, regardless of size, have begun production reductions. Transport being restricted and even confined between countries has further slowed down economic functioning. Most importantly, some panic among consumers and firms have disfigured normal patterns of consumption and caused market inconsistency. Global financial markets have also reacted to changes and global stock market indicators have jumped. ${ }^{1}$

TELEREHABILITATION AND HEALTH CARE AFFECTED BY THE PANDEMIC SITUATION

The clinical infection range appears to have three major patterns: moderate disease with upper respiratory tract symptoms; non-life-threatening pneumonia; and severe pneumonia with Acute Respiratory Distress Syndrome (ARDS) that begins with mild symptoms for 7 - 8 days and then progresses to rapid deterioration and ARDS which requires advance life support. ${ }^{2}$ Patients with transplants and patients suffering from end-stage organ failure are particularly at risk. We are not aware of any gender review of the outbreak in the countries concerned or during the preparatory phases by public health organizations or governments. Although sexdisaggregated COVID-19 evidence shows that same amount of cases is affecting men and women, mortality and disease risk appear to vary. Emerging research proposes that men are dying more than women, possibly because of immunological differences based on gender and patterns such as smoking rates. Understanding the disparity between the influence of pandemic outbreaks on women and men is a crucial phase in understanding the primary and secondary impacts of a health emergency on people and populations and in formulating efficient, equitable strategies and initiatives. ${ }^{3}$ This pandemic has also affected the healthcare department that is treating the patients suffering from diseases other than corona. In several nations, elective operations including live donor transplantation procedures have ceased. ${ }^{4}$ The situation has also seriously affected the field of physical therapy. It was decided by regulatory guidelines that only immediate care would be paid in pandemic duration leaving physiotherapist with the discretionary control in identifying cases that could benefit from their intervention. Though, some understand the importance of physiotherapy some cases may slip into a grey field, leading to asymmetrical access to treatment.

Long term units of care and convalescence are particularly important in sensitive areas. It defines timely approaches in those environments where the primary goal is to reinstate patient activity and quality of life in the short and long term. We should also remember that much of these units' income and social benefit comes from the therapeutic services provided by physical therapists. The sudden loss of income may pose a risk to the institutions' viability. ${ }^{6}$

The question needs to be raised - "Does Covid-19 help the planet work any faster than ever"? The answer to that question is surely "yes". Many physically active services have been discontinued, including outpatient cardiovascular care, school-based physical activity and sports activities, wellness centers and public parks; physical active facilities that was not used by a majority of the global population until COVID-19,7 were reduced considerably. It'd be more plausible to change the name of the COVID-19 pandemic to the sedentarism pandemic at this period. ${ }^{8}$

The COVID-19 pandemic stresses the potential of health services globally, and the delivery of treatment for nonpandemic health problems. ${ }^{9} \mathrm{~A}$ critical feature for monitoring the influx in health services is "reverse triage" - screening patients before they arrive at the Emergency Room (ER). Direct-to-consumer (or on-demand) telemedicine, a proactive triage method of the 21st century that enables patients to be screened effectively, is both patient-centred and conducive to self-quarantine, which prevents patients, physicians, and the environment from infection. It will encourage physicians and patients to connect $24 / 7$, using computers allowed by smartphones or webcams. Respiratory complaints which may be early Covid-19 signs are among the most frequently diagnosed disorders for this method. ${ }^{10}$

Efforts to limit the outbreak shows that social distancing can be successful, at least temporarily, by limiting public transport, border controls and shutting down workplaces as well as mass masking. ${ }^{11}$ In this position it appears that ambulatory and even inpatient recovery plans will be reevaluated. So, how does physiotherapists impact the situation? Logically, if patients and physiotherapy workers are at high risk of exposure to COVID-19, they cannot have in-patient care in addition, because many major health problems, especially in the area of orthopaedics, need rehabilitation and recovery to enhance results. There's an important question here now. In this situation how should doctors approach patients? Homebased recovery and tele-rehabilitation can provide the key, while outpatient, hospitalized, skilled nursing treatment (SNC) for a small number of patients may also be given. ${ }^{12}$

For most home recovery services, tele-rehabilitation is the secret to progress. "Telerehab" is a device that makes the process of healing more available and cost-effective and improves interaction with the subject. Tele-rehabilitation programs use technologies to communicate with remote patients and professionals. Importantly, it will allow better access to rehabilitation care, while access to rehabilitation assessment and treatment these days is challenging. ${ }^{13}$

Tele-health is driven by new technology, which allows one to re-imagine the doctor's appointment as a house call without the ride. For decades the concept of the interactive experience has been around, and telemedicine has been applied across specialties and service lines, from primary care and radiology, cardiology and orthopaedics.

Tele-health is ideally tailored to both primary care and specialist, enabling them to extend their work, serving patients wherever an internet link is accessible. Tele-health projects have historically been operated by a health care network 
because they usually have the resources to purchase massive kiosks of facilities and advanced information technologies.

Tele-rehabilitation is a subset of telemedicine. While this sector is relatively new, its use in developing countries has expanded rapidly. Tele-rehabilitation typically lowers the costs of both health care services and patients relative to conventional inpatient or individual-to-person rehabilitation. In fact, patients living in rural areas where conventional health facilities may not be readily available will benefit from this technology. Nonetheless, some drawbacks to telerehabilitation, including patient cynicism due to remote contact with their doctors or rehabilitators, should not be overlooked. ${ }^{14}$ Tele-rehabilitation has the ability to change health sector and provides people with long-term outpatient therapy. ${ }^{15}$

Various websites, applications and even videoconferencing are used to provide tele-rehabilitation to the needy ones. After the completion of the treatment session a patient can give the feedback in the form of Numerical Pain Rating Scale (NPRS) or Visual Analogue Scale (VAS) which is necessary for the physiotherapist for planning of further sessions. With the help of various applications, which are easily accessible on the smartphones one can improve the fitness and physical activity during this challenging times. Live therapy with the help of videoconferencing can be given to avoid the errors during the treatment; direct monitoring of a patient can be done. Hence it enhances the outcome of the treatment. During the Covid-19 crisis, it will be useful to use instructional videos for patients to monitor their reported videos to ensure that the patient is in compliance with the protocols. ${ }^{13}$ Websites are used for online consultancy. Patient has to register on the website to seek the help, this is sometimes a time consuming process. Smartphone applications are most widely used in recent times as it is not time taking and it also increases the level of treatment for people with disorders in both physical and mental wellbeing. Group therapy or group sessions can be planned for the patients through video calling which can motivate the patient and also is a fun way of treatment for the anxious patients. Exercises can be given in the form of games which could help in the paediatric population. Group Yoga and fitness sessions can be conducted to reduce the effect of this sedentarism pandemic. Online peer review groups not only offer valuable information but also help in most of critical conditions. Meeting other people who go through the same thing will make you feel being less isolated. This will have a positive impact and it can give peace of mind.

Many tele-physiotherapy treatments are administered from a distance without reaching the patient in person, with notable cases of positive urinary stress incontinence care using online assistance and smartphone applications. Tele physiotherapy procedures can provide continuous control of physiological signals in particular populations like cardiorespiratory or orthopaedic illness, such as heart rate, oxygen saturation, electrocardiograms (ECG), and joint motion frequency. Although some tele physiotherapy approaches require custom built instruments, others with off-the-shelf consumer hardware and software have also achieved equally positive outcomes. The pervasive design of the device provides new possibilities for tele physiotherapy, including: tracking of physical activity; sound and light alerts to determine workout speed and duration; real-time input on exercise performance; and text messages to provide guidance or improvement for exercise. Quick web-based diaries for documenting exercise and getting reviews. ${ }^{16}$

Tele-Physio-Therapy (TPT) uses networking tools to promote the administration of patients' physiotherapy inside their own homes either via an audio call or video call. Clinically, TPT provides a variety of recovery and rehabilitation programs including diagnosis, surveillance, detection, care, guidance, instruction, support and counseling. ${ }^{17}$

In order to resolve these issues, TPT should be considered, which includes the usage of telecommunication technologies as a way of supplying information for rehabilitation activities to patients at home who are far from physiotherapy clinics. ${ }^{18}$ The global Covid-19 pandemic forced Brazil's health system to adopt tele-health promptly in different areas of health care. The Federal Council for Physiotherapy and Occupational Therapy (COFFITO) has allowed physiotherapists to use telehealth, in particular tele-consultation and tele-monitoring (Resolution $n^{\circ} 516$ / March 2020). This move was immediately implemented to facilitate secure medical provision as suggested by the WHO. Interactive physical therapy provides benefits for patients, healthcare professionals, and society, such as increasing access to primary care services or clinicians, promoting self-management, improving health care delivery accessibility, and minimizing sick leave length. Any acute and chronic musculoskeletal disorders, heart issues, neurological problems, post-surgical recovery, pain control, pelvic floor injuries, and respiratory dysfunctions have also been investigated. ${ }^{9}$

Tele-rehabilitation helps patients in their own homes to receive online outpatient facilities by videoconferencing with the physiotherapist. Home-based Virtual Reality (VR) equilibrium instruction in patients with Parkinson's Disease (PD) has been shown to minimize postural instability. ${ }^{19}$

A robot-assisted therapy paired with a home exercise plan and a home exercise system alone conducted using a telerehabilitation model can be useful means of enhancing people's quality of life and depression following stroke. ${ }^{20}$

The cardiac tele-rehabilitation program consists of an android-based treatment framework and a web-based portal for caregivers in recovery. The program modules include regular self-assessment, weekly self-assessment, instructional material, expertise in stress and sleep recovery, drug notification, online conversation, prescription food, recommended physical exercise and history of rehabilitation. The modules of self-assessment require users to insert data including physical exercise, diet, blood pressure, heart rate, and body weight. The patient data entries are translated for patients and peers in the program knowledge base and show coloured symbol feedbacks. The mentors review the patient data entries in the dashboard and set out next week's rehabilitation plan. ${ }^{15}$

There is evidence that in multiple orthopaedic patients' home-based therapy is successful, even after significant orthopaedic surgery and serious musculoskeletal problems. A systematic review and meta-analysis was conducted to assess the effectiveness of home-based therapy of patients suffering from hip fracture. Fortunately, home rehabilitation has had an important positive effect on Daily Living Activity (ADL) and physical function in subjects. ${ }^{21}$ 
A well-designed research on the efficacy of the home recovery system for osteoarthritic patients who undergo complete knee arthroplasty also showed positive findings. ${ }^{22}$ Tele-rehabilitation has also been shown as a major replacement for surgical recovery of common musculoskeletal pain. 23

Tele physiotherapy is successful in treating patients with knee osteoarthritis as shown by the study results that demonstrated substantial changes in pain severity and physical activity in patients with knee osteoarthritis after six weeks of tele-physiotherapy. Also, tele-physiotherapy intervention in knee Osteo-Arthritis (OA) patients produces similar pain and physical function outcomes to 'normal' clinical-based physiotherapy where there were no significant differences between tele-physiotherapy group knee osteoarthritis patients and pain intensity in clinical-based physiotherapy group. ${ }^{24}$

Tele-rehabilitation can be used in both the acute COVID-19 intensive treatment phase and in the post-acute period as well. COVID-19 patients may have chronic health issues such as: respiratory; neurological and central nervous system; deconditioning; myopathy up and neuropathy due to serious illness; dysphagia; joint weakness and pain; and psychological problems. ${ }^{25}$ Patients living far from the clinics can find it difficult to visit the clinic regularly because of distance and transportation costs. Some main elements that can be effectively implemented during TPT are self-assessment and self-management, which in turn improves exercise performance and provides improved health results. TPT also helps provide treatments in a timely manner by eliminating hurdles (clinic drive, transit costs, travel time and waiting list), flexible scheduling and expanded patient preference, resulting in a more patient-centered approach to treatment. ${ }^{26}$

Physical therapy or physiotherapy is a primary health care specialty, with physiotherapists supplying patients and communities with resources to create, sustain and improve full lifetime mobility and functional capacity. Physical therapists use the history and physical assessment of a patient to arrive at a diagnosis and to draw up a treatment plan to integrate the findings of diagnostic and imaging tests where appropriate. Electrodiagnostic tests (e.g. electromyograms and tests of the velocity of nerve conduction) can also be useful. Managing physical therapy usually requires administering or helping in individual activities, occupational rehabilitation, schooling and other therapies. Physical therapy has many specialties to name two of the most common ones, including cardiopulmonary, geriatric, neurological, orthopaedic and paediatric. Tele-rehabilitation plays a major part in current physiotherapy work. There are many patients arriving with stroke, for example, from rural areas. Staying in town and getting care was prohibitively expensive to them. Telemedicine has come upon these patients as a great blessing. We will travel to the closest health care facility that has the tele-rehabilitation program option to seek relief for their conditions. Telemedicine \& tele-rehabilitation are valuable methods for the use of information technology in the area of health care (graph published by CIO). Telemedicine \& telerehabilitation is increasing in both developed and developing countries due to the advent of digital services such as telephone and internet in remote and rural areas. E-Medicine is a valuable resource for healthcare practitioners and the general public on different results, information on 14 diseases and treatment available. ${ }^{27}$

Despite some shortcomings, results record substantial and important change in the physical and cognitive health of patients, as well as HRQOL (Health-Related Quality Of Life). Usage of telerehabilitation has been linked with reducing rehabilitative treatment time, cost and inconvenience. High rates of satisfaction indicate the success of the RVTRI (Rural Veterans Tele-Rehabilitation Initiative) in achieving the patient-centred mission. Such findings suggest that in-home video telerehabilitation is a viable alternative to traditional face-to- face recovery therapy. ${ }^{28}$ That form of telemedicine technology is perhaps more critical for health-care officials volunteering in remote locations or serving in the military at present. There aren't many local hospital institutions. Treatment or information may be nearly difficult to obtain. Telemedicine may lead to saving a life.

\section{IMPACT OF COVID 19 PANDEMIC}

\section{Impact of Covid 19 Pandemic on Mental Health} The 2019 corona virus pandemic is a global public health issue and has caused psychological versatility. The outbreak has reflected unfavourable mental health impacts and symptoms. The level of psychological effects such as anxiety, depression and stress since the start of the outbreak has increased due to the sudden shift in scenario, deaths and isolation. ${ }^{29}$ Telehealth, or more specifically tele-mental health facilities, are technically feasible and ideal for helping patients, families and health care professionals during this disease outbreak. ${ }^{30}$ With the help of the telemedicine, group sessions and group activities can be planned to reduce the psychological symptoms of this pandemic. Online seminars and counselling can be provided to patients to reduce the worse stage of the psychological symptoms. Digital peer review communities provide not only useful knowledge but also, more importantly, support. You will feel great, finding other people who are going through the same thing as being less alone. That's going to be positive and it could have peace of mind.

\section{Impact of COVID 19 Pandemic on Economy}

Assessing the economic effects of the COVID-19 pandemic is important for governments, but difficult since the situation has exploded very rapidly. ${ }^{31}$ Whenever a worldwide economic crisis has been reported, the consequences are explicitly observed in the employment process of every country. In the wake of the ongoing pandemic, the impact is predicted to be enormous enough to destroy the existing jobs of thousands of employees in India and this will drive more uncertainty of jobs in the country and globally. Overall, employment levels are predominantly stilled across India's private sector economy, as a slight extension in manufacturing was offset by a small downfall in staffing extent throughout the service sector. The earlier estimates of job data indicate that the corona virus effect may have had a devastating impact on the economy, causing urban unemployment to rise to $30.9 \%$. Global unemployment has risen to $23.4 \%$. Over 50 million people may have lost their jobs within two weeks of lockdown. 


\section{Impact of COVID 19 Pandemic on Mode of Transfer}

Travel industry is distinctly one of the worst-hit sectors and the ban on international flights has all but shut down this fragment entirely. The main reasons for the increasing pandemic risk in the 21st century are: rapidly growing and dispersed world population; trends in urbanization and population concentration; centralized food production in global supply chains; expanded usage of higher-order foods such as meat; and expansion of global transport networks that serve as vectors for the spread of pathogens. ${ }^{32}$ As the trouble in sectors such as aviation and hospitality are extensively discussed, there are many travel related industries such as foreign exchange, which are even more affected than aviation and hotels, and are frequently overlooked entirely. Due to the shutdown of international and domestic travel, demand for the turbine fuel will comparatively decline. Indian Association of Tour Operators (IATO) and the World Travel and Tourism Council (WTTC) forecasts that hospitality, aviation and travel sector together may suffer loss of billions of rupees, resulting in a loss of half a billion of jobs due to travel restrictions being forced on foreign tourists. Lockdown to curb the spread of the corona virus, which has stalled all businesses in India, barring essential services, the real estate sector counts on top, such as the half constructed building, metros and big cities making the land ineffectual and workers jobless.

\section{Impact of COVID 19 Pandemic on Source of Energy \\ COVID-19 has disrupted some of the farming and supply} chains operations. The Food and Agriculture Organization reports that COVID-2019 impacts agriculture in two critical aspects: availability and demand for food. These two things are closely linked to food health, and thus food protection is also at risk. ${ }^{33}$ Primary reports show that some harvesting activities are interrupted by the unavailability of migrant laborers, particularly in the northwest zone where wheat and pulses are harvested. Supply chains interrupt due to distribution challenges and other concerns. Prices for wheat, vegetables and other commodities have fallen but customers still spend more. The shutdown of hotels, restaurants, sweet shops and tea shops depresses the dairy sales. Meanwhile, owing to misguidance, especially because of social media, poultry farmers have been badly hit after the spread of news that chickens are the COVID-19 carriers.

\section{Impact of COVID 19 Pandemic on Education System}

The reaction to the COVID-19, school closing at national level as an immediate measure to avoid infection transmission, was mandated and the immediate home-schooling program was strictly enforced. Although the new closures of schools vary in that schooling is supposed to begin online, closures are likely to deepen the achievement divide among children between low-income and higher-income families. Children from lowincome households live in situations that render home education challenging because electronic learning environments typically include computers and a safe internet connection. ${ }^{34}$

Impact of COVID 19 Pandemic on Environment The impacts have been positive for the environment. The COVID-19 increased China's air quality in the near term and leads greatly to a worldwide cut in carbon release. 35 The quarantine time has resulted in lesser use of single-use plastics, which can find their way into our water bodies and has reduced water pollution, although the effects are only temporary.

To be well prepared is the most important aspect for the future pandemic. ${ }^{36}$ In crucial phases like these it is vital to be informed and important for every individual to unfold the accurate and right facts for the advancement of individuals in the society. ${ }^{37}$ Pollution has remarkable reduced on Earth. Which is beneficial not only for the human beings but for each and every life on the planet.

The COVID-19 corona virus crisis affects different sectors in different ways. Production, transportation, and distribution based companies are the worst-hit. It revels the weaknesses of business firms that requires physical presence and manual processes. Against this background, we expect a raise for digitalization and automation in the long term. Those companies that have already digitized and automated the majority of their processes are already in a good situation. Likewise, companies that have made progress in shifting to cloud-based delivery are now satisfying from the scalability of the performance and flexibility of costs. As an economic impact, psychological hit due to pandemic has its count; specific somatic effects were correlated with a considerable psychological impact of the outbreak in females and students.

\section{CONCLUSIONS}

Thus, as the world scrambles to ease the immense healthcare load of the virus, most economies are bracing for the havoc, the virus will leave behind. Many countries have already announced some rounds of 'economic packages' to help businesses, workers and healthcare systems engulfed by the crisis. The Indian government and RBI have also put in place a slew of measures to help fight the corona virus disease hazards and improve its economic downfall. Healthcare is also affected, as a result of the social distancing to break the chain of infection, and patients are unable to visit the clinics and hospitals. Digitalization and automation have proved to be the solution in this challenging phase. Telemedicine and tele physiotherapy with the help various smartphone applications and websites for online consultancy have changed the time in a positive way. Conducting live sessions whether it may be individual, or group sessions reduces the psychological risks to the patients and also is beneficial for the motivation of the therapy. Thus, telemedicine and tele physiotherapy increase the level of treatment for people with disorders for both physical and mental wellbeing, lower the hospital expenses, strengthen conventional face-to-face practice, and boost treatment adherence and satisfaction. 
Financial or other competing interests: None.

Disclosure forms provided by the authors are available with the full text of this article at jemds.com.

PP and SJ contributed to the creation and design of the study. SJ and PP wrote the manuscript of the article. All the authors read and approved the final manuscript for publication

\section{REFERENCES}

[1] McKibbin W, Fernando R. The global macroeconomic impacts of COVID-19: Seven scenarios. CAMA Working Papers. Centre for Applied Macroeconomic Analysis, Crawford School of Public Policy, The Australian National University; 2020 Mar [cited 2020 Jun 6]. (CAMA Working Papers). Report 2020-19. https://ideas.repec.org/p/een/camaaa/2020-19.html

[2] COVID-19: What is next for public health? - The Lancet (cited 2020 Jun 6]. https://www.thelancet.com/journals/lancet/article/PII S0140-6736(20)30374-3/fulltext?hss_channel=tw27013292

[3] Wenham C, Smith J, Morgan R, et al. CovID-19: the gendered impacts of the outbreak. Lancet 2020;395(10227):846-8.

[4] Ahn C, Amer H, Anglicheau D, et al. Global Transplantation COVID Report March 2020. Transplantation 2020:10.1097/TP.0000000000003258.

[5] Physiotherapy management for COVID-19 in the acute hospital setting: clinical practice recommendations ScienceDirect [cited 2020 Jun 17]. https://www.sciencedirect.com/science/article/pii/S18 3695532030028X

[6] Alpalhão V, Alpalhão M. Impact of COVID-19 on physical therapist practice in Portugal. Phys Ther 2020: pzaa071.

[7] WHO. Physical inactivity: a global public health problem. [cited 2020 Jun 17]. https://www.who.int/dietphysicalactivity/factsheet_ina ctivity/en/

[8] Hall G, Laddu DR, Phillips SA, et al. A tale of two pandemics: How will COVID-19 and global trends in physical inactivity and sedentary behavior affect one another? Prog Cardiovasc Dis 2020:S00330620(20)30077-3.

[9] Dantas LO, Barreto RPG, Ferreira CHJ. Digital physical therapy in the COVID-19 pandemic. Braz J Phys Ther 2020.

[10] Hollander JE, Carr BG. Virtually perfect? Telemedicine for Covid-19. N Engl J Med 2020;382(18):1679-81.

[11] Wang G, Zhang Y, Zhao J, et al. Mitigate the effects of home confinement on children during the COVID-19 outbreak. The Lancet 2020;395(10228):945-7.

[12] Hollander JE, Carr BG. Virtually perfect? Telemedicine for Covid-19. N Engl J Med 2020;382(18):1679-81.

[13] Azhari A, Parsa A. Covid-19 outbreak highlights: importance of home- based rehabilitation in orthopedic surgery. Arch Bone Jt Surg 2020;8(Suppl 1):317-8.

[14] Peretti A, Amenta F, Tayebati SK, et al. Telerehabilitation: review of the state-of-the-art and areas of application. JMIR Rehabil Assist Technol 2017;4(2):e7.

[15] Jameie S, Haybar H, Aslani A, et al. Development and usability evaluation of web-based telerehabilitation platform for patients after myocardial infarction. Stud Health Technol Inform 2019;261:68-74.

[16] Holland AE. Telephysiotherapy: time to get online. Journal of Physiotherapy 2017;63(4):193-5.

[17] Odole AC, Ojo OD, Odunaiya NA, et al. Tele-physiotherapy in Nigeria: perceived challenges by physiotherapists to its implementation. International Journal of Telemedicine and Clinical Practices 2015;1(2):186 https://www.inderscienceonline.com/doi/abs/10.1504/IJ TMCP.2015.069763

[18] Bernard MM, Janson F, Flora PK, et al. Videoconferencebased physiotherapy and tele-assessment for homebound older adults: a pilot study. J Activities, Adaptation \& Aging 2009;33(1):39-48.

https://www.tandfonline.com/doi/abs/10.1080/0192478 0902718608

[19] Gandolfi M, Geroin C, Dimitrova E, et al. Virtual reality telerehabilitation for postural instability in Parkinson's disease: a multicenter, single-blind, randomized, controlled trial. Biomed Res Int 2017;2017:7962826.

[20] Linder SM, Rosenfeldt AB, Bay RC, et al. Improving quality of life and depression after stroke through telerehabilitation. Am J Occup Ther 2015;69(2):6902290020p1-10.

[21] Desheng W, Xiulan Z, Shaowel Z. Effect of home-based rehabilitation for hip fracture: a meta-analysis of randomized controlled trials. Ingenta Connect: J Rehabil Med 2018;50(6):481-6.

[22] Donghai L, Zhouyuan Y, Pengde K, et al. Home-based compared with hospital-based rehabilitation program for patients undergoing total knee arthroplasty for osteoarthritis: a systematic review and meta-analysis of randomized controlled trials. Am J Physical Med \& Rehabil 2017;96(6):440-7.

[23] Mbada C, Olaoye M, Ayanniyi O, et al. Comparative efficacy of clinic-based and telerehabilitation application of Mckenzie therapy in low-back pain. Archives of Physical Medicine and Rehabilitation 2017;98(10):E46-E7.

[24] Odole AC, Ojo OD. A telephone-based physiotherapy intervention for patients with osteoarthritis of the knee. Int J Telerehabil 2013;5(2):11-20.

[25] Summary-Evidence-Telerehabilitation-post-acute-COVID19.pdf [cited 2020 Jun 29]. https://www.lenus.ie/bitstream/handle/10147/627810/S ummary-Evidence-Telerehabilitation-post-acute-COVID19.pdf? sequence $=1$

[26] Adhikari SP, Shrestha P, Dev R. Feasibility and effectiveness of telephone-based telephysiotherapy for treatment of pain in low-resource setting: a retrospective pre-post design. Pain Research and Management 2020;2020:2741278.

[27] Application-Of-Telemedicine-E-Medicine-InPhysiotherapy-Emerging-Trends.Pdf [cited 2020 Jun 30].https://www.researchgate.net/profile/Drramakrishna n_Ks/publication/331276971_application_of_telemedicine _e_medicine_in_physiotherapy emerging_trends/links/5c6fd776299bf1268d1bfbd7/appli cation-of-telemedicine-e-medicine-in-physiotherapyemerging-trends.pdf

[28] Levy CE, Silverman E, Jia H, et al. Effects of physical therapy delivery via home video telerehabilitation on functional and health-related quality of life outcomes. J Rehabil Res Dev 2015;52(3):361-70. 
[29] Wang C, Pan R, Wan X, et al. Immediate psychological responses and associated factors during the initial stage of the 2019 Coronavirus Disease (COVID-19) Epidemic among the general population in China. Int J Environ Res Public Health 2020;17(5):1729.

[30] Zhou X, Snoswell CL, Harding LE, et al. The role of telehealth in reducing the mental health burden from COVID-19. Telemedicine J E Health 2020;26(4):377-9.

[31] Baker SR, Bloom N, Davis SJ, et al. COVID-Induced Economic Uncertainty. National Bureau of Economic Research: 2020 Apr [cited 2020 Jun 6]. (Working Paper Series). $\quad$ Report No.: 26983. http://www.nber.org/papers/w26983

[32] Gössling S, Scott D, Hall CM. Pandemics, tourism and global change: a rapid assessment of COVID-19. Journal of Sustainable Tourism 2020 Apr 27. [cited 2020 Jun 6]. https://doi.org/10.1080/09669582.2020.1758708

[33] Siche R. What is the impact of COVID-19 disease on agriculture? Scientia Agropecuaria 2020;11(1):3-6. http://www.scielo.org.pe/scielo.php?script=sci_abstract \&pid=S2077-

$99172020000100003 \& \operatorname{lng}=e s \& n r m=i s o \& \ln g=e n$
[34] COVID-19, school closures and child poverty: a social crisis in the making - The Lancet Public Health [cited 2020 Jun 6]. https://www.thelancet.com/journals/lanpub/article/PII S2468-2667(20)30084-

$0 /$ fulltext?utm_campaign=updatelanpub\&utm_source $=\mathrm{h}$ s_email\&utm_medium $=$ email\&utm_content $=88236179 \&$ _hsenc=p2ANqtz-cC608_HyGN0KJgZaaKJrwZXAP2_ucPCPTyvdNdARaqiW qRb9Z-CfycUZmgdbV1XIe1DVK3qqw088wbTyqR9uYgFme1iABnJXJRc9A AWLCKJ4PauvGU4FtccJ0f_EqL4aV64D\&_hsmi=8823617 9

[35] Wang Q, Su M. A preliminary assessment of the impact of COVID-19 on environment - a case study of China. Science Total Environment 2020;728:138915.

[36] Shah P, Naqvi W. Fighting and chasing the rogue virusCovid 19. International Journal of Research in Pharmaceutical Sciences 2020;11(SPL1):77-80.

[37] Dutta S, Acharya S, Shukla S, et al. COVID-19 pandemicrevisiting the myths. Int J Med Sci 2020;7(5):7-10. 